\title{
Spinal cord MRI in clinically isolated optic neuritis
}

\section{M Dalton, P A Brex, K A Miszkiel, K Fernando, D G MacManus, G T Plant, A J Thompson, D H Miller}

Background/methods: One hundred and fifteen patients with clinically isolated optic neuritis underwent magnetic resonance imaging (MRI) of the brain and spinal cord within 3 months of the onset of symptoms.

Results: Eighty one (70\%) patients had brain lesions and 31 $(27 \%)$ had cord lesions. Cord lesions were seen in $12 \%$ with a normal brain MRI, $21 \%$ with between one and eight brain lesions, and $45 \%$ with nine or more brain lesions. When the new diagnostic criteria for MS were applied, MRI cord imaging used for evidence of dissemination in time and space allowed a diagnosis of MS in only one additional asymptomatic patient at 1 year, two additional asymptomatic patients at 3 years.

Conclusions: Using existing criteria, spinal cord imaging rarely contributes to the diagnosis in patients with clinically isolated optic neuritis.

U sing the new diagnostic criteria formulated by an international panel of experts, both brain and spinal cord lesions seen on magnetic resonance imaging (MRI) scans may be used to diagnose multiple sclerosis (MS) in patients with clinically isolated optic neuritis. ${ }^{1}$ While spinal cord imaging is essential when the clinically isolated syndrome involves the spinal cord to exclude other diagnoses, its value in diagnosis of patients with optic neuritis has not been systematically investigated. In recent years, we have been prospectively recruiting patients with isolated optic neuritis for investigation with brain and spinal cord MRI. The purpose of this study was to document the frequency of MRI abnormalities in both regions, and to determine their potential to enable a diagnosis of MS, and to predict the future clinical course. We now report the findings in a cohort of 115 patients with optic neuritis who have had both brain and spinal cord imaging at presentation, and have been followed up for periods ranging from 0 to 72 months (mean 18 months).

\section{METHODS}

Design of the prospective clinically isolated syndrome follow up study

Since 1995, patients between 16 and 50 years of age with optic neuritis have been recruited from the physicians' clinic at Moorfields Eye Hospital. Approval was received from the medical ethics committee. Consent was obtained from all patients before entry into the study. Inclusion in the study was based solely on clinical features and was not influenced by MRI. Patients with a previous history of possible demyelinating events were excluded. Baseline clinical and MRI assessments were performed less than 3 months from clinical onset. Appropriate investigations were carried out to exclude alternative diagnoses.

\section{MRI acquisition protocols}

Brain MRI was performed at baseline and again after 3 months, 1 year, and 3 years. Images were acquired using a 1.5 Tesla Signa imager (General Electric, Milwaukee, WI, USA). Each patient was given $0.1 \mathrm{mmol} / \mathrm{kg}$ of gadoliniumDTPA prior to imaging.

Proton density (PD) and T2 weighted images were acquired using a dual echo, fast spin echo (FSE) sequence with a repetition time (TR) of $3200 \mathrm{~ms}$, and effective time (TE) of 15/90 ms. A Tl weighted spin echo image (TR $600 \mathrm{~ms}$, TE $14 \mathrm{~ms}$ ) of the brain was acquired $15 \mathrm{~min}$ after injection of gadolinium-DTPA. For each sequence, $46 \times 3 \mathrm{~mm}$ contiguous axial slices were acquired. The field of view was $24 \mathrm{~cm}$, matrix was $256 \times 256$, and number of excitations was 1 .

MRI of the spinal cord was performed at baseline, 1 year, and 3 years. Nine contiguous, $3 \mathrm{~mm}$ thick sagittal slices were obtained through the whole of the spinal cord with PD and T2 weighted FSE (TR $2500 \mathrm{~ms}$, TE 56/98 ms), and T1 weighted spin echo (TR $500 \mathrm{~ms}$, TE $19 \mathrm{~ms}$ ) sequences following administration of gadolinium-DTPA. For both sequences, phased array coils and a $48 \mathrm{~cm}$ field of view were employed, with a $512 \times 512$ matrix (fig 1).

\section{Clinical assessment}

Patients were assessed clinically at baseline, 3 months, 1 year, and 3 years. Those who experienced further relapses with new signs were classified as having clinically definite MS according to the criteria of Poser. ${ }^{2}$ MS was diagnosed using the new McDonald criteria from clinical and/or MRI evidence of dissemination in time and space. Disability was measured using the Kurtzke Expanded Disability Status Scale (data not shown). ${ }^{3}$

\section{Image analysis}

The images were reviewed by an experienced neuroradiologist, who was blinded to the clinical diagnosis. The number and location of high signal $\mathrm{PD} / \mathrm{T} 2$ lesions, and gadolinium enhancing Tl weighted lesions was noted. The baseline, 3 month, 1 year, and 3 year proton density/T2 weighted, and gadolinium enhanced Tl weighted scans were analysed for evidence of dissemination in space and time using the McDonald criteria. Dissemination in space, based on the criteria of Barkhof and Tintore, ${ }^{45}$ consisted of three out of four of the following: $(a)$ at least one gadolinium enhancing or nine T2 lesions; $(b)$ at least one infratentorial lesion; $(c)$ at least one juxtacortical lesion; $(d)$ at least three periventricular lesions.

The criteria allowed the replacement of a brain lesion with a cord lesion in any of the above categories. One or more

Abbreviations: FSE, fast spin echo; $M R I$, magnetic resonance imaging; $M S$, multiple sclerosis; PD, proton density; TE, effective time; TR, repetition time 


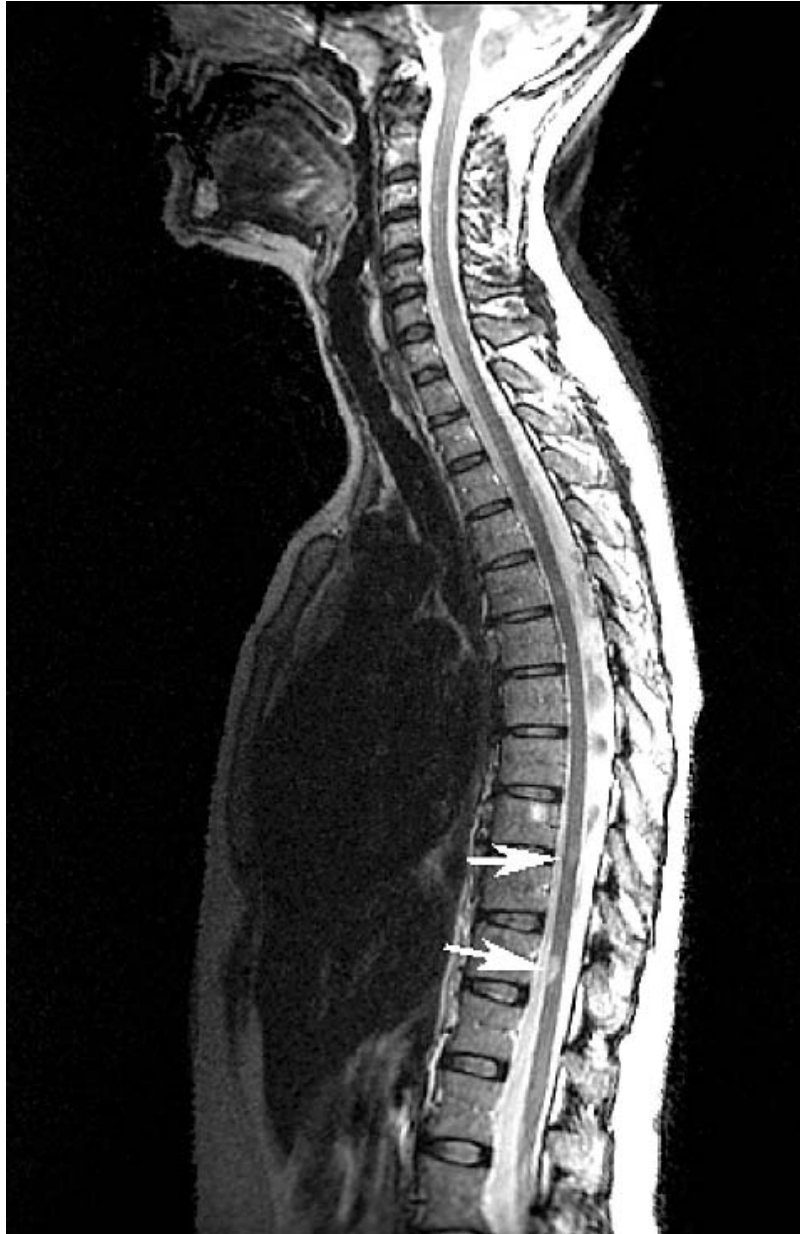

Figure 1. T2 weighted sagittal MRI through the spinal cord in a 30 year old female with isolated optic neuritis. Two intrinsic lesions are seen in the thoracic cord (arrowed).

gadolinium enhancing lesions at 3 months (brain only) or a new T2 weighted lesion on the 1 or 3 year follow up scans (brain and spinal cord) was used as evidence of dissemination in time.

Patients were also divided into those with no brain lesions, those with 1-8 brain lesions inclusive, and those with nine or more brain lesions, and these subgroups were subsequently classified for MRI evidence of dissemination in space.

\section{RESULTS}

\section{Baseline clinical data}

One hundred and fifteen patients with optic neuritis had imaging of the brain and spinal cord within 3 months of the onset of symptoms: 113 had unilateral and two had bilateral optic neuritis (age: (mean, median, range) 32, 31, 16-49 years, gender: 76F, 39M, and disease duration: 6, 5, 1-12 weeks).

\section{MRI dissemination in space: baseline data}

Eighty one $(70 \%)$ patients had one or more brain lesions and $31(27 \%)$ had one or more cord lesions. Forty one/115 (36\%) had dissemination in space using brain scanning alone (44/ 115 (38\%) using brain and spinal cord) (table 1).

\section{Normal brain MRI}

Of the 34 patients with a normal brain MRI scan, four (12\%) had spinal cord lesions. Using the present diagnostic criteria, patients with cord lesions but without MRI, brain lesions cannot be included as having MRI evidence of dissemination in space.
1-8 brain lesions

Of the 39 patients with between one and eight lesions, eight (21\%) had spinal cord lesions. The number of patients with dissemination in space on the baseline scan was increased by the inclusion of spinal cord lesions from two (5\%) to five (13\%). Lesion location in the three patients requiring cord lesions for dissemination in space was: patient 1: periventricular (3), gadolinium enhancing (1), cord (1); patient 2: infratentorial (1), juxtacortical (2), periventricular (2), and cord (3); and patient 3: infratentorial (2), periventricular (3), cord (2).

Lesion location in the 34/39 patients with between one and eight lesions who did not have dissemination in space despite inclusion of the spinal cord was: cord (4), infratentorial (5), juxtacortical (15), periventricular (19), periventricular $\geqslant 3$ (6), discrete cerebral white matter neither periventricular nor juxtacortical (14), and gadolinium enhancing (6).

Nine or more brain lesions

In the 42 patients with nine or more brain lesions, 19 (45\%) had spinal cord lesions. On brain MRI alone, 39/42 (93\%) had dissemination in space, and this number was unchanged when cord MRI findings were incorporated.

When multiple cord lesions were allowed to substitute for an identical number of brain lesions, there was no alteration in the number of patients who had dissemination in space. Only two patients with more than one cord lesion lacked dissemination in space at baseline.

\section{Follow up: MRI dissemination in time and space, and diagnosis of MS}

The follow up study is ongoing, and the present report includes all those currently followed up at each time point: 94 have been studied at 3 months, 64 at 1 year (of whom one did not have cord MRI), and 44 at 3 years (of whom three did not have cord MRI). Although the new diagnostic criteria incorporating brain MRI findings markedly increase the number of patients with a diagnosis of MS at 3 months, 1 year, and 3 years, when compared with the number developing clinically definite MS, imaging of the spinal cord had almost no additional impact on the diagnosis. As spinal cord imaging was not performed at 3 months, the impact of new spinal cord lesions on dissemination in time could only be evaluated at 1 and 3 years. Overall, addition of cord MRI findings to brain MRI and clinical findings enabled a diagnosis of MS using the McDonald criteria in only $1 / 64$ (1.6\%) and $2 / 44(4.5 \%)$ additional patients who were followed up at 1 and 3 years respectively. These individuals all had between one and eight brain lesions at baseline (table 1).

At 1 year, only $1 / 11$ patients with new spinal cord lesions did not have clinical (new relapse) or brain MRI (new brain lesion) evidence of dissemination in time; this single patient had a normal brain scan at baseline and 1 year. One further patient with a new brain lesion at 1 year and cord lesion at baseline had MRI evidence of dissemination in space according to the McDonald criteria only if the spinal cord lesion was included (lesions: periventricular (4), juxtacortical (2), discrete (1), and cord (1)).

At three years, 2/10 patients with new spinal cord lesions did not have MRI evidence of dissemination in space, although both had new brain lesions and no further symptoms.

In patients with an abnormal baseline scan, the occurrence of new T2 brain lesions as evidence of dissemination in time at 3 months or 1 year had a sensitivity of $85 \%$ and specificity of $79 \%$ for clinically definite MS at 3 years. Adding new cord lesions at 1 year resulted in no increase in sensitivity, and specificity dropped from $79 \%$ to $75 \%$ (table 2 ). 
Table 1 Impact of spinal cord lesions on the diagnosis of MS

\begin{tabular}{|c|c|c|c|c|c|}
\hline Time & Patient characteristics & No. of patients & 0 brain lesions & 1-8 brain lesions & $\geqslant 9$ brain lesions \\
\hline \multirow[t]{4}{*}{ Baseline } & Patients with brain lesions (cord) & 115 & $34(4)$ & $39(8)$ & $42(19)$ \\
\hline & Patients with cord lesions & 115 & $12 \%$ & $21 \%$ & $45 \%$ \\
\hline & Mean (range) cord lesions & 115 & $0.1(0-1)$ & $0.4(0-4)$ & $1.2(0-11)$ \\
\hline & DIS: brain, brain+cord & 115 & 0 & $2(5 \%), 5(13 \%)$ & $39(93 \%), 39(93 \%)$ \\
\hline \multirow[t]{4}{*}{3 months } & New brain lesions & 94 & $1 / 28(4 \%)$ & $8 / 32(25 \%)$ & $23 / 34(68 \%)$ \\
\hline & McDonald MS (brain only) & 94 & $0 / 28$ & $1 / 32(3 \%)$ & $17 / 34(50 \%)$ \\
\hline & McDonald MS (brain+ (baseline only) cord) & ) 94 & $0 / 28$ & $1 / 32(3 \%)$ & $17 / 34(50 \%)$ \\
\hline & Clinically definite MS & 94 & $0 / 28$ & $0 / 32$ & $9 / 34(26 \%)$ \\
\hline \multirow[t]{6}{*}{1 year } & New brain lesions & 64 & $0 / 17$ & $11 / 22(50 \%)$ & $18 / 25(72 \%)$ \\
\hline & New cord lesions & 63 & $2 / 17$ & $3 / 22(14 \%)$ & $6 / 24(25 \%)$ \\
\hline & New brain or cord lesions & 64 & $2 / 17(12 \%)$ & $11 / 22(50 \%)$ & $19 / 25(76 \%)$ \\
\hline & McDonald MS (brain only) & 64 & $1 / 17(6 \%)$ & $7 / 22(32 \%)$ & $22 / 25(88 \%)$ \\
\hline & McDonald MS (brain and cord) & 64 & $1 / 17(6 \%)$ & $8 / 22(36 \%)$ & $22 / 25(88 \%)$ \\
\hline & Clinically definite MS & 64 & $1 / 17(6 \%)$ & $1 / 22(5 \%)$ & $8 / 25(32 \%)$ \\
\hline \multirow[t]{6}{*}{3 years } & New brain lesions & 44 & $3 / 15(20 \%)$ & $11 / 15(73 \%)$ & $12 / 14(86 \%)$ \\
\hline & New cord lesions & 41 & $1 / 15(7 \%)$ & $4 / 14(29 \%)$ & $5 / 12(42 \%)$ \\
\hline & New brain or cord lesions & 44 & $3 / 15(20 \%)$ & $11 / 15(73 \%)$ & $13 / 14(93 \%)$ \\
\hline & McDonald MS (brain only) & 44 & $1 / 15(7 \%)$ & $5 / 15(33 \%)$ & $14 / 14(100 \%)$ \\
\hline & McDonald MS (brain and cord) & 44 & $1 / 15(7 \%)$ & $7 / 15(47 \%)$ & $14 / 14(100 \%)$ \\
\hline & Clinically definite MS 3 years & 44 & $1 / 15(7 \%)$ & $4 / 15(27 \%)$ & $9 / 14(64 \%)$ \\
\hline
\end{tabular}

\section{DISCUSSION}

Patients with clinically isolated optic neuritis and asymptomatic MRI brain lesions have an increased risk for the development of MS. $^{6}$ Although spinal cord imaging is mandatory in isolated spinal cord syndrome patients, to detect treatable alternative disorders such as compressive lesions, its role is unclear in patients with optic neuritis. The key question is whether cord lesions increase the frequency of dissemination in space and time, and thereby enable a more frequent and earlier diagnosis of MS.

Overall, 27\% of our patients had MRI evidence of spinal cord lesions at presentation. However, they contributed little to the diagnosis. Although four patients had cord lesions but no brain lesions, these subjects did not have dissemination in space according to current definitions, and therefore a diagnosis of MS cannot be made using existing MRI criteria. Two of these four have been followed for 3 years and have had no further symptoms.

In patients with between one and eight brain lesions inclusive, cord imaging modestly increased the number of patients with dissemination in space at baseline by $8 \%$; however, at 3 months (the earliest time point at which a diagnosis of MS can be made) no additional patients were diagnosed with MS (although follow up MRI was confined to the brain at this time point). In those patients with nine or more brain lesions, cord lesions were much more common, being found in almost one half of subjects, but their presence did not increase the number who fulfilled the MRI criteria for dissemination in space or who developed MS at each follow up time point.

The present study is limited by the fact that cord imaging was not performed at 3 months. Nevertheless, brain MRI, on average, detects many more lesions than cord MRI, and the latter is most often abnormal only when there are at least nine brain lesions already present and when the new criteria for dissemination in space are very often already fulfilled by brain findings alone. In established MS, new cord lesions occur less frequently on follow up than new brain lesions ${ }^{78}$ and usually when there are concurrent new brain lesions. Notably, the new spinal cord lesions seen at 3 years compared with 1 year did not increase the number of patients exhibiting dissemination in time over that period, and increased the number of patients with a diagnosis of MS at 3 years by only two, out of a total of $44(4.5 \%)$ (table 1 ). We doubt therefore that additional cord imaging at month 3 would have affected the rate of early MS diagnosis.
Table 2 Relationship of new lesions after 1 year with clinically definite MS after 3 years in patients with an abnormal baseline MRI brain (41 patients)

\begin{tabular}{lcc}
\hline & New brain lesions** & $\begin{array}{l}\text { New brain and } \\
\text { cord lesions* }\end{array}$ \\
\hline ClS+ & 6 & 7 \\
MS+ & 11 & 11 \\
CIS- & 22 & 21 \\
MS- & 2 & 2 \\
Sensitivity (95\% CI) & $85 \%(55$ to $98 \%)$ & $85 \%(55$ to $98 \%)$ \\
Specificity (95\% Cl) & $79 \%(59$ to $92 \%)$ & $75 \%(55$ to $89 \%)$ \\
PPV (95\% CI) & $65 \%(38$ to $86 \%)$ & $61 \%(36$ to $83 \%)$ \\
NPV (95\% CI) & $92 \%(73$ to $99 \%)$ & $91 \%(72$ to $99 \%)$ \\
Accuracy (95\% Cl) & $80 \%(65$ to $91 \%)$ & $78 \%(62$ to $89 \%)$ \\
\hline
\end{tabular}

$\mathrm{Cl}$, confidence interval.

CIS+, new lesions after 1 year, and clinically isolated syndrome after 3 years; MS+, new lesions after 1 year, and clinically definite MS after 3 years; CIS-, no new lesions after 1 year, and clinically isolated syndrome after 3 years; MS-, no new lesions after 1 year, and multiple sclerosis after 3 years.

*Brain MRI findings only were available at 3 months; brain and cord MRI findings were available at 1 year.

Based on the new MRI lesions after 1 year as the test, and clinically definite MS at 3 years as the disease, the number of true positives (TP; new MRI lesions positive, clinically definite MS positive), true negatives (TN; new MRI lesions negative, clinically definite MS negative), false positives (FP; new MRI lesions positive, clinically definite MS negative), and false negatives (FN; new MRI lesions negative, clinically definite MS positive) were calculated and used to determine:

- sensitivity: probability of the test finding disease among those who have disease (TP/TP+FN)

- specificity: probability of test finding no disease among those who do not have disease (TN/TN+FP)

- positive predictive value: percentage of people with a positive test result who have the disease (TP/TP+FP)

- negative predictive value:percentage of people with a negative test result who do not have disease (TN/TN+FN)

- accuracy $=$ proximity to the true value $(T P+T N / T P+T N+F N+F P)$.

In summary, using existing criteria, imaging of the spinal cord is of limited diagnostic value in patients with optic neuritis with between one and eight MRI brain lesions. If in future the diagnostic criteria were revised to allow the presence of any cord lesions as evidence of dissemination in space and time, then cord imaging would have a diagnostic role in more patients, but overall it appears that this will be a relatively small subgroup. Any such extension of the diagnostic criteria should first be validated by confirming its accuracy for predicting clinically definite MS. 


\section{ACKNOWLEDGMENTS}

We thank Dr J I O'Riordan for his assistance in this study, Dr D Altmann for his help with the statistical analysis and Miss C Middleditch for her technical assistance. C M Dalton was sponsored by Élan and K Fernando was sponsored by Biogen. Schering AG sponsored P A Brex. The NMR Unit is supported by a program grant from the Multiple Sclerosis Society of Great Britain and Northern Ireland.

\section{Authors' affiliations}

C M Dalton, P A Brex, K Fernando, D G MacManus, A J Thompson,

D H Miller, NMR Research Unit, Institute of Neurology, London, UK

K A Miszkiel, Lysholm Department of Neuroradiology, National Hospital for Neurology and Neurosurgery, London, UK

G T Plant, Moorfields Eye Hospital, London, UK

Competing interest: none declared

Correspondence to: Professor D H Miller, NMR Research Unit, Department of Neuroinflammation, Institute of Neurology, University College London, Queen Square, London WCIN 3BG, UK d.miller@ion.ucl.ac.uk

\section{REFERENCES}

1 McDonald WI, Compston A, Edan G, et al. Recommended diagnostic criteria for multiple sclerosis: guidelines from the International Panel on the diagnosis of multiple sclerosis. Ann Neurol 2001;50:121-7.

2 Poser CM, Paty DW, Scheinberg L, et al. New diagnostic criteria for multiple sclerosis: guidelines for research protocols. Ann Neurol 1983:13:227-31.

3 Kurtzke JF. Rating neurologic impairment in multiple sclerosis: an Expanded Disability Status Scale (EDSS). Neurology 1983;33:1444-52.

4 Barkhof F, Filippi M, Miller DH, et al. Comparison of MRI criteria at first presentation to predict conversion to clinically definite multiple sclerosis. Brain 1997: 120:2059-69

5 Tintore M, Rovira A, Martinez MJ, et al. Isolated demyelinating syndromes: comparison of different MR imaging criteria to predict conversion to clinically definite multiple sclerosis. Am J Neuroradiol 2000;21:702-6.

6 Brex PA, Ciccarelli O, O'Riordan J, et al. A longitudinal study of abnormalities on MRI and disability from multiple sclerosis. N Engl J Med 2002;346:158-64

7 Thorpe JW, Kidd D, Moseley IF, et al. Spinal MRI in patients with suspected multiple sclerosis and negative brain MRI. Brain 1996;119:709-14.

8 Silver NC, Good CD, Sormani MP, et al. A modified protocol to improve the detection of enhancing brain and spinal cord lesions in multiple sclerosis. J Neurol 2001;248:215-24.

\section{$\mathrm{ECHO}$}

\section{Family shares a new nystagmus locus}

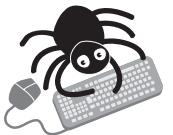

Please visit the Journal of Neurology, Neurosurgery, and Psychiatry website [www.jnnp. com] for a link to the full text of this article. genetic study of one family may improve our understanding of early eye stabilisation $\triangle$ and squint because it suggests that their control may lie in single gene. Linkage - analysis for nystagmus showed linkage with markers between 13q31-q33, not previously reported. This defines the dominantly inherited familial disorder as dominant acquired vestibulocerebellar disorder, not a benign form of cerebellar ataxia.

In four generations of the family affected members had gaze evoked nystagmus developing at 1 to 2 years of age. It was non-progressive and ataxia, if present, was minimal; there were no abnormalities on neuroimaging. Squint was also present and more common in family members with nystagmus, and much more so than in the general population. Vision was preserved except for family members with amblyopia related to squint. No corresponding structural anomalies have been identified that would explain the functional disorder. The researchers speculate that a failure in function of the flocculus may be involved

The study included 30 family members examined by an ophthalmologist, orthoptist, and eye movement specialist. DNA from each was used in a genomic screen against 400 polymorphic repeat microsatellite markers and 17 other markers in pairwise linkage analysis.

This form of early acquired nystagmus, which is not congenital, has nor been investigated before.

A Journal of Medical Genetics 2003;40:37-41. 